\title{
Concomitant Agent
}

National Cancer Institute

\section{Source}

National Cancer Institute. Concomitant Agent. NCI Thesaurus. Code C70902.

A pharmaceutical agent other than agent(s) tested in a given protocol that is administered to or used by a subject either prior to or during the study. Supportive care and essential ancillary medications required by a treatment regimen should be clearly identified. A protocol complete instructions including appropriate indication, dosage, administration route, schedule, restrictions to use, and any other relevant data should be explicitly stated. Concept also refers to substance(s) used prior or during the study that are not specified by the protocol. 